\title{
Intoxicação espontânea e experimental por Pterodon emarginatus (Fabaceae Faboideae) em bovinos e experimental em ovinos ${ }^{1}$
}

\author{
Raquel A.S. Cruz ${ }^{2}$, Leonardo P. de Oliveira ${ }^{3}$, Flavio H.B. Caldeira², Fábio S. Mendonça ${ }^{4}$, \\ Flávia B. Bacha ${ }^{5}$, Arnildo Pott ${ }^{6}$, Ricardo A.A. Lemos ${ }^{5}$ e Edson M. Colodel ${ }^{7 *}$
}

\begin{abstract}
Cruz R.A.S., Oliveira L.P., Caldeira F.H.B., Mendonça F.S., Bacha F.B., Pott A., Lemos R.A.A. \& Colodel E.M. 2012. [Spontaneous and experimental poisoning by Pterodon emarginatus (Fabaceae Faboideae) in cattle and experimental poisoning in sheep.] Intoxicação espontânea e experimental por Pterodon emarginatus (Fabaceae Faboideae) em bovinos e experimental em ovinos. Pesquisa Veterinária Brasileira 32(11):1087-1094. Departamento de Clínica Médica Veterinária, Faculdade de Agronomia, Medicina Veterinária e Zootecnia, Universidade Federal de Mato Grosso, Av. Fernando Corrêa da Costa 2367, Bairro Boa Esperança, Cuiabá, MT 78068-900, Brazil. E-mail: moleta@ufmt.br

This paper reports cattle mortalities in the states of Mato Grosso and Mato Grosso do Sul, Brazil, caused by the consumption of the leaves of Pterodon emarginatus. The outbreaks occurred during the dry season after P. emarginatus trees had fallen to the ground due to high winds or after having been cut for wood, and cattle had access to the branches. Forty cattle died during three different outbreaks. First clinical signs were seen after 24-72 hours of the consumption of the leaves by the cattle. The course of the letal disease was from 12 to 36 hours. Clinical signs were characterized by apathy, depression, walking aimlessly, pressing the head against objects, and occasionally photosensitization in cases of poisoning with a longer clinical course. The disease was reproduced by administration of $P$. emarginatus leaves at a minimum toxic dose of $20 \mathrm{~g} / \mathrm{kg}$ for sheep and $6 \mathrm{~g} / \mathrm{kg}$ for cattle. The main macroscopic findings in spontaneous and experimental cases were in the liver, characterized by hepatomegaly and diffuse accentuation of the lobular pattern on the capsular and cut surfaces. Additionally, there were petechiae, ecchymoses and suffusions within the thoracic and abdominal serosa surfaces. In two outbreaks, fotossensibilization was reported in cattle that survived the acute phase. The main histopathological findings were marked centrilobular or massive coagulative hepatocellular necrosis associated with congestion and hemorrhages, surrounded or not by markedly swollen and vesicular hepatocytes in the periacinar area. The diagnosis was based on epidemiological data, clinical signs, necropsy findings, histological lesions and experimental reproduction of the poisoning in cattle and sheep. These findings showed P. emarginatus to be a hepatotoxic plant of interest in the Midwest region of Brazil.
\end{abstract}

INDEX TERMS: Poisonous plants, Pterodon emarginatus, Fabaceae Faboideae, plant poisoning, cattle, sheep.

RESUMO.- Relatam-se mortalidades de bovinos nos Estados de Mato Grosso e Mato Grosso do Sul relacionadas ao consumo de folhas de Pterodon emarginatus. Três surtos

\footnotetext{
${ }^{1}$ Recebido em 17 de abril de 2012.

Aceito para publicação em 27 de junho de 2012.

${ }^{2}$ Programa de Pós-Graduação em Ciências Veterinárias, Faculdade de Agronomia, Medicina Veterinária e Zootecnia (Famev), Universidade Federal de Mato Grosso (UFMT), Av. Fernando Corrêa da Costa 2367, Bairro Boa Esperança, Cuiabá, MT 78068-900, Brasil.

${ }^{3}$ Curso de Medicina Veterinária, Famev, Universidade Federal de Mato Grosso, Cuiabá, MT.

${ }^{4}$ Departamento de Morfologia e Fisiologia Animal, Universidade Fede-
}

ocorreram no período de seca na região Centro-Oeste, após a queda de árvores devido a ventos fortes ou após serem derrubadas para aproveitamento da madeira. Morreram

\footnotetext{
ral Rural do Pernambuco (UFRPE), Rua Dom Manuel Medeiros s/n, Dois Irmãos, Recife, PE 52171-900, Brasil.

${ }^{5}$ Faculdade de Medicina Veterinária e Zootecnia (Famez), Universidade Federal do Mato Grosso do Sul (UFMS), Cx. Postal 649, Campo Grande, MS 79070-900, Brasil.

${ }^{6}$ Herbário GGMS, Departamento de Biologia, UFMS, Cidade Universitária s/n, Cx. Postal 549, Campo Grande, MS 79070-900.

${ }^{7}$ Departamento de Clínica Médica Veterinária (Climev), Famev-UFMT, Cuiabá, MT. *Autor para correspondência: moleta@ufmt.br
} 
40 bovinos em três diferentes surtos. A intoxicação foi reproduzida com administração de folhas de P. emarginatus; a dose tóxica mínima foi $20 \mathrm{~g} / \mathrm{kg}$ para ovinos e $6 \mathrm{~g} / \mathrm{kg}$ para bovinos. Os sinais clínicos iniciaram-se entre 24 e 72 horas após o consumo da planta. A evolução clínica da doença letal foi de 12 a 36 horas. Os sinais clínicos se caracterizaram por apatia, depressão, andar a esmo, pressão da cabeça contra objetos. Em dois dos surtos, os bovinos que sobreviveram a fase aguda da doença, desenvolveram fotossensibilização. Os principais achados macroscópicos nos casos espontâneos e experimentais foram no fígado; estes se caracterizaram por hepatomegalia e evidenciação do padrão lobular na superfície capsular e de corte. Notaram-se também hemorragias nas serosas abdominais e torácicas. Microscopicamente observou-se necrose coagulativa hepatocelular, que variou de centrolobular a massiva, por vezes associada à congestão e hemorragia; ocorreu também marcada tumefação e vacuolização de hepatócitos na região periacinar. 0 diagnóstico foi baseado nos dados epidemiológicos, clínicos, patológicos e na reprodução experimental da intoxicação em bovinos e ovinos. Estes achados caracterizam P. emarginatus como planta hepatotóxica de interesse pecuário na região Centro-Oeste do Brasil.

TERMOS DE INDEXAÇÃO: Plantas tóxicas, Pterodon emarginatus, Fabaceae Faboideae, intoxicação por plantas, bovinos, ovinos.

\section{INTRODUÇÃO}

0 gênero Pterodon pertence à família Fabaceae (=Leguminosae), subfamília Faboideae (=Papilionoideae); é composto por quatro espécies arbóreas nativas brasileiras: Pterodon abruptus Benth., P. emarginatus Vogel (sin. Pterodon pubescens), P. apparicioli Pedersoli e P. polygalaeflorus Benth. (Carvalho 2004). Essas espécies são encontradas em terrenos secos e arenosos do cerrado dos estados de Tocantins, Mato Grosso, Mato Grosso do Sul, Goiás e áreas de transição para florestas semidecíduas nos Estados de Minas Gerais e São Paulo (Carvalho 2004, Lorenzi \& Matos 2008).

Pterodon emarginatus, popularmente conhecida como sucupira, faveiro, sucupira-branca e sucupira-lisa, é uma árvore com altura de $8-16 \mathrm{~m}$, decídua, intolerante ao sombreamento, e que floresce nos meses de setembro e outubro, com frutificação em outubro a novembro (Lorenzi 2002). Devido ao seu uso na medicina popular brasileira suas atividades farmacológicas anti-inflamatórias, analgésicas e anti-reumáticas têm sido amplamente estudadas (Carvalho et al. 1999, Sabino et al. 1999b, Coelho et al. 2005, Dutra et al. 2009b)

Estudos fitoquímicos com espécies do gênero Pterodon revelaram a presença de alcalóides triterpenóides nas cascas (Bustamante et al. 2010), isoflavonas e triterpenos nos caules (Marques et al. 1998), diterpenos (Fascio et al. 1975, Arriaga et al., 2000) e isoflavonas (Braz \& Gottlieb 1971) nas sementes e os fitoesteróides $\beta$-sitosterol e estigmasterol nas folhas das plantas (Santos et al. 2010). Apesar de várias pesquisas terem demonstrado os efeitos fitoterápicos de espécies de Pterodon, estudos envolvendo a descrição dos efeitos tóxicos dessas plantas são escassos. Em um trabalho realizado com camundongos submetidos a um trata- mento dérmico com óleo de sucupira (Luiz Dias 1995), os animais não apresentaram sinais de intoxicação, porém a injeção de doses de 0,5 e 1,0mL do óleo das sementes de Pterodon emarginatus induziu efeitos tóxicos. Em outro estudo comprovou-se a ação genotóxica de P. emarginatus em linfócitos T humanos (Machado et al. 2011).

O objetivo deste trabalho é descrever os aspectos clínicos e anatomopatológicos da intoxicação espontânea por P. emarginatus em bovinos nos Estados de Mato Grosso e Mato Grosso do Sul e a reprodução experimental da intoxicação em bovinos e ovinos.

\section{MATERIAL E MÉTODOS}

\section{Intoxicação espontânea}

Relatam-se três surtos de mortalidade de bovinos após o consumo espontâneo das folhas de Pterodon emarginatus (Fig.1). Dois destes ocorreram no Estado de Mato Grosso, o primeiro (S1) no município de Cáceres em setembro de 2005 e o outro (S2) no município de Barra do Bugres em outubro de 2007; o terceiro ocorreu no Estado de Mato Grosso do Sul no município de Dois Irmãos do Buriti em setembro de 2006 (S3).

Os bovinos do S1 foram necropsiados por Médicos Veterinários do Instituto de Defesa Agropecuária do Estado de Mato Grosso (Indea-MT) e os bovinos do S2 pela equipe do Laboratório de Patologia Veterinária da Universidade Federal de Mato Grosso (LPV-UFMT). As amostras dos órgãos de ambos os surtos foram encaminhadas em formol a 10\% para o LPV-UFMT. Os bovinos do S3 foram necropsiados pelo Médico Veterinário da propriedade, que encaminhou as amostras em formol a $10 \%$ para o Laboratório de Anatomia Patológica Animal da Universidade Federal de Mato Grosso do Sul (LAP-UFMS). Os dados clínicos e epidemiológicos dos três surtos foram obtidos através de entrevistas com médicos veterinários, proprietários e vaqueiros das respectivas propriedades.

\section{Coleta e identificação botânica}

As amostras de folhas de Pterodon emarginatus foram coletadas em quatro momentos. Na Coleta 1 em outubro de 2007, coletaram-se folhas das árvores derrubadas nos piquetes onde ocorreu a mortalidade de bovinos dois dias após a ocorrência do S2. A Coleta 2 foi realizada em outubro 2010, na propriedade do S3. A Coleta 3 foi feita em outubro de 2011, às margens da rodovia MT-246 (S15․07'.31.3" e W056 56'.62.2') na localidade de Raizama, município de Barra do Bugres, MT. Nas Coletas 2 e 3, as folhas foram secas à sombra, separada dos talos, trituradas e mantidas refrigeradas. 0 peso das folhas secas representou $50 \%$ das folhas verdes. Encaminharam-se para a identificação botânica, amostras de plantas do S1 e S2 ao Herbário Central da UFMT e amostras do S3 para o Herbário da UFMS. A Coleta 4 ocorreu em março de 2008 na Fazenda Mangueira Nova, Alto Paraguai, MT; esta última foi usada na intoxicação experimental do Bovino 1.

\section{Intoxicação experimental em ovinos}

Realizaram-se experimentos em dois locais para confirmação da toxicidade da planta. A metodologia empregada foi semelhante em ambos. Cinco ovinos (Quadro 1) tiveram jejum sólido de 24 horas e hídrico de 8 horas antes do início do experimento. Posteriormente recebiam feno de alfafa, silagem de milho e água $\mathrm{ad}$ libitum além de porções diárias de 100 a $200 \mathrm{~g}$ de ração comercial. Os ovinos eram clinicamente acompanhados a cada hora após a observação de alterações clínicas.

No LPV-UFMT utilizaram-se três fêmeas da raça Santa Inês pesando 40, 35 e 27,7 Kg, respectivamente, que permaneceram 


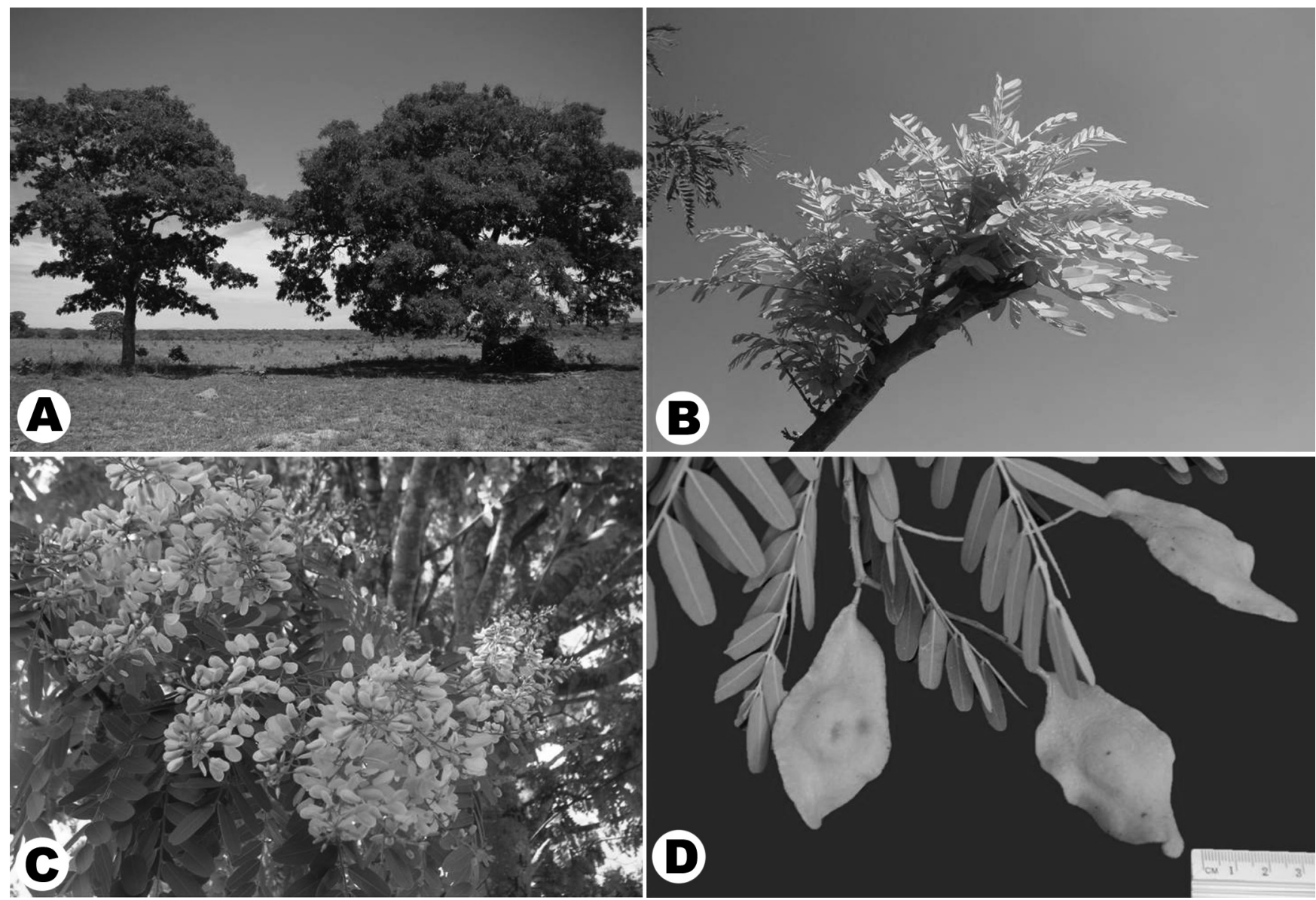

Fig.1. (A) Pterodon emarginatus, Município de Cáceres, MT, novembro de 2009. (B) Brotação de folhas, setembro de 2009, Município de Cáceres, MT. (C) inflorescência em setembro, 2009. Município de Alto Paraguai, MT. (D) Favas de P. emarginatus em novembro de 2009, Município de Alto Paraguai, MT.

\section{Quadro 1. Delineamento experimental da intoxicação por folhas de Pterodon emarginatus em ovinos}

\begin{tabular}{|c|c|c|c|c|c|}
\hline Ovinos & 1 & 2 & 11 & 31 & 10 \\
\hline Raça & Santa Inês & Santa Inês & Cruzado & Cruzado & Santa Inês \\
\hline Sexo & Fêmea & Fêmea & Macho & Macho & Fêmea \\
\hline Peso (kg) & 40 & 35 & 38,5 & 35,8 & 27,7 \\
\hline Local de Coleta & Coleta $1^{\mathrm{a}}$ & Coleta $1^{\text {a }}$ & Coleta $2^{\text {b }}$ & Coleta $2^{\text {b }}$ & Coleta $3^{c}$ \\
\hline Local do Experimento & LPV/UFMT & LPV/UFMT & LAP/UFMS & LAP/UFMS & LPV/UFMT \\
\hline Conservação das folhas de P. emarginatus & Frescas $^{\mathrm{d}}$ & Frescas & Secas & Secas $^{d}$ & Secas \\
\hline Forma de administração & Oral forçada & Oral forçada & Fístula ruminal & Fístula ruminal & Sonda esôgafica \\
\hline Dose $\mathrm{g} / \mathrm{kg}$ & 20 & 15 & 20 & 20 & 15 \\
\hline Número de administrações (por dia) & 1 & 1 & 1 & 1 & 1 \\
\hline Evolução clinica (horas) & 24 & - & 48 & 72 & 24 \\
\hline Desfecho & Morreu & Recuperou & Morreu & Morreu & Morreu \\
\hline
\end{tabular}

a Coleta em Barra do Bugres outubro 2007, b Coleta UFMS, c Coleta MT-246, Raizama, Barra do Bugres, outubro de 2011.

em baias individuais. Duas receberam por via oral forçada, $20 \mathrm{~g} /$ $\mathrm{kg}$ e $15 \mathrm{~g} / \mathrm{kg}$ de folhas frescas (Coleta 1 ) e a terceira recebeu por sonda esofágica $15 \mathrm{~g} / \mathrm{kg}$ de folhas secas (equivalente a $30 \mathrm{~g} / \mathrm{kg}$ de folhas verdes) (Coleta 3). No LAP-UFMS utilizaram-se dois ovinos machos da raça Santa Inês, pesando 38,5 e 35,8 kg; estes receberam $20 \mathrm{~g} / \mathrm{kg}$ de folhas secas de $P$. emarginatus (Coleta 2) através de fístula ruminal.

\section{Intoxicação experimental em bovinos}

Foram usados 3 bovinos em três locais diferentes como descrito no Quadro 2.

No LPV da Universidade de Cuiabá (Unic), em janeiro de 2008, o Bovino 1, macho, cruzado, 2,5 anos de idade, mantido em baia individual, recebeu por via oral forçada doses fracionadas em três dias consecutivos que totalizaram $12 \mathrm{~kg}(25 \mathrm{~g} / \mathrm{kg})$ das folhas frescas de Pterodon emarginatus (Coleta 4). Adicionalmente recebeu feno de alfafa, ração comercial e água ad libitum. Este bovino foi clinicamente acompanhado e após 48 horas da administração da planta foi submetido a biópsia hepática, através da técnica que utiliza a agulha de Menghini (Braga et al. 1985, Barros et al., 2007).

No LAP-UFMS, em agosto de 2011, o Bovino 2, fêmea, 1 ano, Nelore, $153 \mathrm{~kg}$, mantido em baia individual, recebeu 20g/ kg das folhas secas de $P$. emarginatus (Coleta 2), fornecidas através de fístula ruminal. Posteriormente recebeu feno de alfafa e água $\mathrm{ad}$ libitum. Foram acompanhados os parâmetros clínicos diariamente. 
Quadro 2. Delineamento experimental da intoxicação por folhas de Pterodon emarginatus em bovinos

\begin{tabular}{lccc}
\hline \multicolumn{1}{c}{ Bovino } & 1 & 2 & 56 \\
\hline Raça & Cruzado & Cruzado & Caracu \\
Sexo & Macho & Fêmea & Macho \\
Peso (kg) & 380 & 153 & 300 \\
Local de Coleta & Alto Paraguai & Dois Irmãos do Buriti & Barra do Bugres \\
Local do Experimento & LPV/UNIC & LAP/UFMS & LPV/UFMT \\
Conservação das folhas de P. emarginatus & Frescas & Secas & Secas \\
Forma de administração & Oral forçada & Fístula ruminal & Sonda esofágica \\
Dose g/kg & 25 & 20 & 3 \\
Número de administrações (por dia) & 1 & 1 & 1 \\
Evolução (Dias) & 30 & 3 & 8 \\
Desfecho & Recuperou & Recuperou & Morreu
\end{tabular}

Na Fazenda Experimental da UFMT, em outubro de 2011, o Bovino 3, macho, raça caracu, com peso de $320 \mathrm{~kg}$, recebeu $3 \mathrm{~g} /$ kg de folhas secas de P. emarginatus (Coleta 3) por sonda esofágica, adicionada a 25 litros de água. Posteriormente foi solto em piquete com pasto nativo para acompanhamento clínico, recebeu também água e silagem de milho ad libitum. Os parâmetros clínicos eram avaliados diariamente.

\section{Análise histopatológica}

Tanto nos casos espontâneos quanto nos experimentais amostras de órgãos como encéfalo, fígado, pulmão, coração, rim, intestino, linfonodos, baço, rúmen, retículo e abomaso foram colhidos durante as necropsias e os fragmentos de fígado, obtidos por biópsia do Bovino 1, foram fixados em formalina $10 \%$. As amostras foram processadas rotineiramente, coradas pela Hematoxilina e Eosina (HE) e analisadas em microscópio óptico (Prophet 1992).

\section{RESULTADOS}

Aspectos epidemiológicos, clínicos e patológicos da intoxicação natural em bovinos

Surto 1. Propriedade localizada no município de Cáceres no Estado de Mato Grosso, com criação extensiva de bovinos de corte. No dia 10 de setembro de 2005 após uma forte ventania, caíram várias árvores de Pterodon emarginatus (Fig.2), que estavam em período de rebrota das folhas e em floração, em 3 piquetes diferentes da propriedade. A mortalidade se iniciou no dia 12 de setembro de 2005 e estendeu-se por 3 dias. Em um dos piquetes morreram

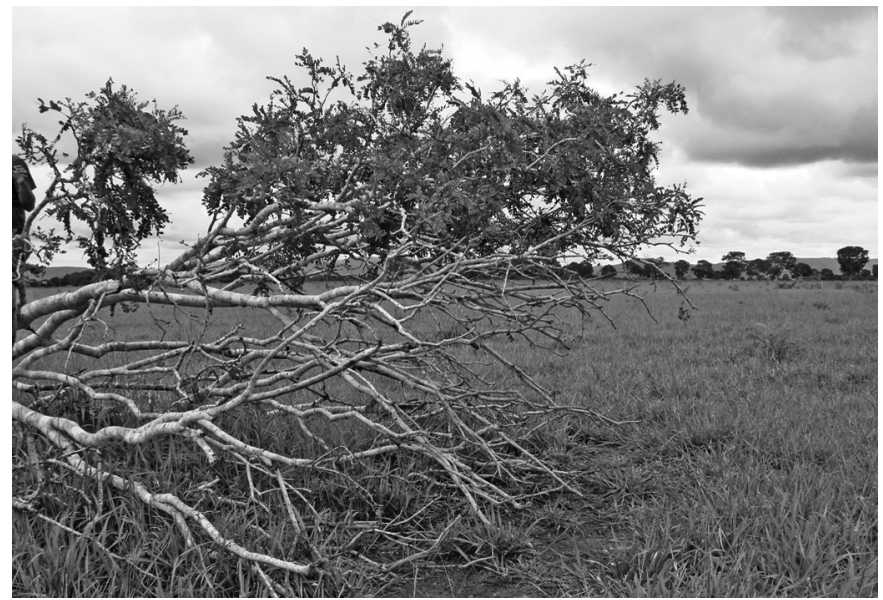

Fig.2. Pterodon emarginatus, arvore caída em área de pastoreio, com sinais de consumo pelos bovinos. Setembro de 2005, Cáceres, MT.
$4 / 12$, em outro $2 / 20$ e num terceiro $26 / 150$ bovinos. A evolução clínica da doença foi de 12 a 36 horas e os principais sinais clínicos relatados foram desidratação, alteração de comportamento com apatia ou agressividade, inquietação, fezes ressecadas, decúbito esternal, decúbito lateral e morte.

Um bovino necropsiado tinha hemorragias nas serosas torácicas e abdominais, fígado com manchas amareladas e padrão lobular aumentado. Também se notou vesícula biliar distendida com conteúdo fluido esverdeado. Relatou-se que alguns bovinos tiveram quadro de fotossensibilização e morreram, mas não foram necropsiados.

Surto 2. Propriedade localizada no município de Barra do Bugres-MT, com criação extensiva de bovinos de corte. No mês de setembro de 2007, 80 vacas foram colocadas em um piquete de 30 hectares de Brachiaria brizantha. Os animais recebiam suplementação com ração comercial, sal mineral em cocho e água em um açude. No mês de outubro duas árvores de P. emarginatus foram derrubadas para produção de mourões de cerca e os galhos e folhas ficaram na pastagem e foram consumidos pelos bovinos. Após 3 dias da derrubada das árvores, 3 bovinos apresentaram sinais clínicos neurológicos com tremores, andar cambaleante, cegueira com andar a esmo e choques em obstáculos; a evolução clínica foi de 12 a 24 horas. Passados dois dias após a ocorrência da mortalidade, um quarto bovino apresentou marcada apatia, anorexia, inquietação, corrimento ocular seroso, fotofobia e prurido, e após uma semana, acentuado quadro de fotossensibilização associado à mumificação da pele escrotal, prepucial e perineal, com áreas irregulares de necrose do muflo nasal e da mucosa nasal e oral, inclusive superfície ventral da língua. Havia também marcada icterícia e a urina tinha coloração vermelha-acastanhada. Este bovino foi eutanasiado in extremis após 15 dias de evolução clinica da doença.

Nos três bovinos necropsiados constataram-se extensas hemorragias no tecido subcutâneo e nas serosas abdominais (Fig.3), o fígado estava aumentado, friável, com evidenciação do padrão lobular na superfície capsular e de corte, e com áreas vermelho-escuras intercaladas com áreas claras. Havia também manchas irregulares amareladas principalmente na face diafragmática. A vesícula biliar estava repleta e notava-se edema da parede. Petéquias, equimoses e sufusões foram observadas no tecido subcutâneo, nas serosas abdominais, superfície pleural visceral e parietal, pe- 

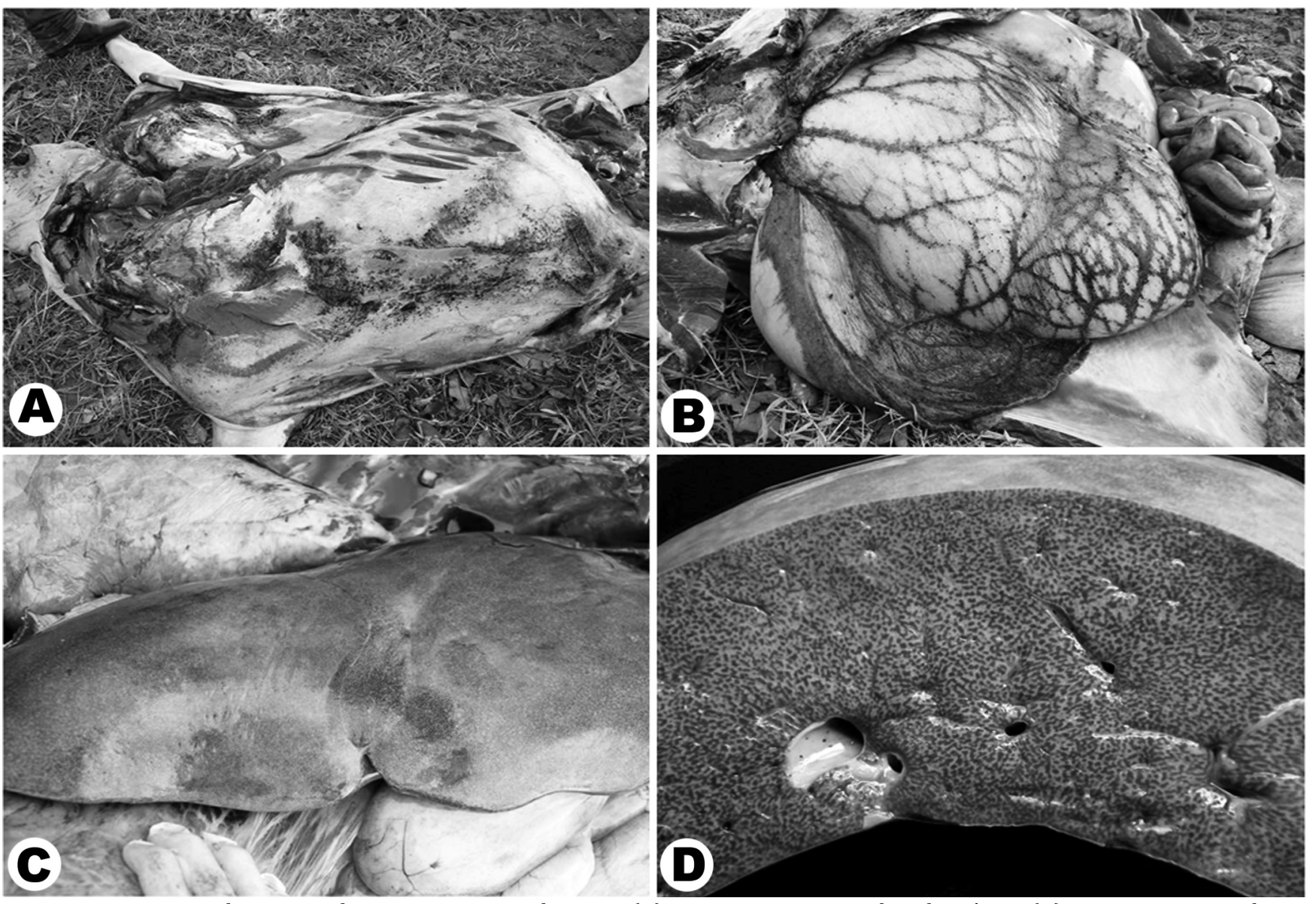

Fig.3. Intoxicação natural por Pterodon emarginatus em bovinos. (A) Hemorragias no tecido subcutâneo, (B) no omento, nos sulcos ruminais, na serosa intestinal e (C) aumento do padrão lobular do fígado na superfície capsular e (D) de corte.

ricárdio e endocárdio. No intestino havia edema de parede e as fezes na ampola retal estavam ressequidas e revestidas por muco e sangue. Na necropsia do bovino com fotossensibilização, além das alterações observadas durante o exame clínico, havia icterícia acentuada e generalizada. 0 fígado estava aumentado, com coloração amarela-alaranjada e a vesícula biliar repleta. Os rins tinham coloração castanha-escura na superfície subcapsular e ao corte.

Surto 3. Ocorreu no município de Dois Irmãos do Buriti/MS no mês de setembro de 2006, em uma propriedade de criação extensiva de gado de corte. Os animais repentinamente apresentaram sintomatologia nervosa que se caracterizava por incoordenação motora dos membros posteriores, além de fezes de coloração enegrecida. Cinco animais ficaram doentes; eram quatro vacas prenhes e um bezerro desmamado; todos morreram. Um bovino foi necropsiado; observaram-se fígado aumentado de volume, com padrão lobular aumentado e áreas amarelo-claras intercalas com áreas vermelho-enegrecidas. Notou-se também mucosa ocular hemorrágica e líquido de coloração vermelho escuro na cavidade abdominal.

\section{Achados microscópicos na intoxicação espontânea em bovinos}

A principal alteração observada nos bovinos dos três surtos foi necrose coagulativa hepatocelular que variava de centrolobular a massiva, caracterizada por dissociação dos cordões de hepatócitos com aumento do espaço canalicular e de Disse, além de marcada congestão e hemorragias (Fig.4). Os hepatócitos necróticos estavam diminuídos em volume, com contornos irregulares, o núcleo frequentemente estava picnótico ou cariorrético e o citoplasma eosinoflílico ou vacuolizado. Em alguns bovinos que tinham

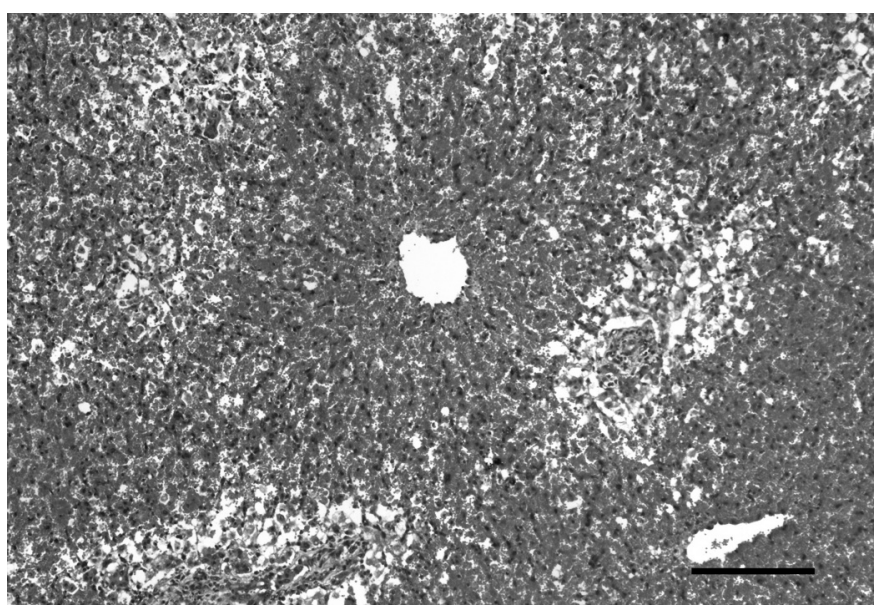

Fig.4. Intoxicação natural por Pterodon emarginatus em bovinos. Fígado com necrose coagulativa hepatocelular centrolobular associada com congestão e hemorragia e acentuada e tumefação de hepatócitos na região periacinar. HE, obj.10x. 
padrão de necrose centrolobular, notava-se acentuada tumefação e vacuolização macrovacuolar de hepatócitos das regiões mediozonal e periacinar. No fígado e linfonodos de bovinos do S1 e S2 havia ocasionalmente células espumosas mono ou multinucleadas isoladas ou em agregados aleatórios, além de pericolangite mononuclear discreta ou leve. No bovino do S1 notou-se vacuolização do neurópilo (Status spongiosus) principalmente no pedúnculo cerebelar e na substancia branca das folhas cerebelares. No bovino do S2 com fotossensibilização, notou-se intensa tumefação hepatocelular, colestase e necrose individual de hepatocitos, além de leve pericolangite mononuclear. Nas áreas de pele com fotossensibilização havia necrose coagulativa epidermal, com infiltrados neutrofílicos multifocais e detritos celulares (microabscessos epidermais), hiperqueratose peraqueratótica, acantose irregular, tumefação epidermal com desorganização epidermal (edema) e acantólise aleatória. Frequentemente se observava crostas superficiais com detritos celulares, fibrina, neutrófilos e miríades de bactérias coco-bacilares. Na derme notou-se intenso edema e infiltrado perivascular misto.

\section{Intoxicação experimental em ovinos}

0 Ovino 1, após 18 horas da administração da planta, apresentou apatia, anorexia, salivação, icterícia leve de mucosas, cegueira aparente com ausência de reflexo palpebral, fraqueza com tremores musculares, principalmente de pescoço e membros posteriores quando em estação, pressão da cabeça contra a parede da baia e andar a esmo. Notou-se também atonia ruminal, decúbito lateral com a cabeça voltada para a grade costal (pleurotono), frequentemente com plano nasal apoiado no solo, incapacidade de permanecer em estação mesmo com auxilio, após 12 horas de evolução. Posteriormente o ovino ficou em decúbito lateral com movimentos de pedalagem, coma e morte após 17 horas de evolução clínica.

No Ovino 2 notaram-se alterações clínicas após 14 horas da administração da planta, que foram caracterizadas por anorexia, depressão moderada, permanência em estação em posição de cavalete, com cabeça baixa, sialoréia moderada, corrimento nasal mucoso e tremores musculares de cabeça e dos membros posteriores. Houve diminuição do volume de fezes, que inicialmente eram pastosas com muco; 24 horas após havia predomínio apenas de muco. Após 30 horas da evolução da doença, o ovino aparentou melhora clínica e após 4 dias da administração da planta, começou a se alimentar e foi considerando clinicamente recuperado.

O Ovino 10, oito horas após da administração da planta, apresentou abdome distendido, apatia e letargia, dificuldade respiratória com taquipnéia e respiração ofegante; permaneceu em decúbito esternal com pleurotono e posteriormente em decúbito lateral com pedaleio, e morreu após 24 horas.

Os Ovinos 11 e 31 apresentaram apatia, anorexia e ficavam com a boca e plano nasal na lâmina da água do cocho, porém não bebiam água. Os Ovinos 11 e 31 morreram 24 e 48 horas, respectivamente, após a $2^{\underline{a}}$ administração da planta.
Os achados de necropsia em todos os ovinos foram uniformes e consistiram em fígado aumentado de volume, difusamente amarelo-pálido, friável e com leve aumento do padrão lobular na superfície capsular e de corte. No parênquima, havia áreas vermelho-escuras intercaladas com áreas amarelo-claras distribuídas difusamente. No rúmen, era possível observar grande quantidade de folhas de $P$. emarginatus. Havia também hiperemia da mucosa intestinal; as fezes se encontravam ressecadas e continham muco. Microscopicamente as lesões hepáticas foram caracterizadas por necrose hepatocelular coagulativa. Os Ovinos $\mathbf{1}$ e 10 apresentaram necrose massiva, desorganização e tumefação de hepatócitos remanescentes próximo ao espaço porta, congestão severa, hemorragia e pericolangite mononuclear leve. A necrose hepática nos Ovino 11 e 31 era predominantemente centro-lobular com acentuada tumefação periacinar, e havia presença de glóbulos eosinofílicos além de congestão e hemorragia. Nos Ovinos 2 e 10 havia células espumosas aleatoriamente distribuídas, em agregados ou isoladas, ocasionalmente multinucleadas, no fígado e em linfonodos hepático e mesentéricos.

\section{Intoxicação experimental em bovinos}

O Bovino 1 apresentou sinais clínicos a partir de 192 horas da administração da planta. Os sinais clínicos se caracterizaram por moderada apatia, letargia, anorexia, desidratação, lacrimejamento, mucosas conjuntivais congestas e hipotonia dos movimentos ruminais. Após 288 horas o bovino apresentava-se levemente ictérico, com andar cambaleante e trôpego; passadas 336 horas, o bovino permanecia por longos períodos em decúbito esternal, apático e só ficava em estação quando insistentemente auxiliado. Após 576 horas essas alterações tornaram-se menos intensas, com progressivo aumento do apetite, melhora da marcha e com 720 horas o bovino havia se recuperado da doença. 0 Bovino 2, após o consumo da planta, não apresentou sinais clínicos aparentes. O Bovino 56, após 24 horas da administração da planta, apresentou-se levemente apático e não respondia ao ser tangido. Após 96 horas estava com acentuada apatia, mucosas ictéricas, edema de prepúcio, letargia e leve dismetria. Passada 120 horas apresentava-se com acentuada depressão e leve incoordenação. As mucosas estavam ictéricas, a região nasal estava tumefeita e com discreta exsudação serosa que evoluiu para estriações que variavam de avermelhada a alaranjadas; ocasionalmente notavam-se crostas amarronzadas com moderada exsudação serosa amarelada nas regiões de pele clara. A pele do pescoço estava ressecada e a do prepúcio e escroto com coloração enegrecida. Notaram-se alterações de comportamento com marcada agressividade e incoordenação motora após 136 horas do consumo. Este bovino entrou em um açude e não conseguiu sair, e morreu após 168 horas da administração da planta; foi retirado do açude para necropsia 12 horas após a morte. Na necropsia havia hemorragias de serosas, o fígado estava moderadamente aumentado, com padrão lobular evidente e havia repleção da vesícula biliar e edema de alças intestinais.

$\mathrm{Na}$ análise histológica dos tecidos coletados nas biopsias do Bovino 1 foi observada necrose hepatocelular cen- 
trolobular discreta e tumefação mediozonal e periacinar, difusa e acentuada. No Bovino 56, as alterações relacionadas à autólise impediram a análise histológica.

\section{DISCUSSÃO}

O diagnóstico da intoxicação natural por Pterordon emarginatus baseou-se nos dados epidemiológicos e sinais clínicos, e na ocorrência de necrose coagulativa hepatocelular, observada nos três surtos. Além disso, o diagnóstico foi confirmado com a reprodução do quadro clínico e lesional através da administração de folhas de $P$. emarginatus tanto em ovinos quanto em bovinos.

Esse quadro é similar ao descrito em outras intoxicações por plantas que ocorrem no estado de Mato Grosso do Sul e que causam necrose hepática em bovinos, que são as intoxicações por Cestrum laevigatum (Purisco et al. 1998), Vernonia rubricaulis (Brum et al. 2002) e Vernonia mollissima (Döbereiner et al. 1976). Essas mortalidades geralmente ocorrem em pastagens degradadas, com rebrota dessas plantas e são associadas à fome dos bovinos (Purisco et al. 1998, Tokarnia et al. 2000).

Durante o desenvolvimento desse estudo notou-se que os bovinos, mesmo demonstrando avidez inicial no primeiro contato com as folhas, rapidamente rejeitam seu consumo. Os casos com mortalidade relatados neste trabalho ocorreram durante o período de seca na região Centro-Oeste, entre os meses de setembro e outubro, período no qual ocorrem ventanias e chuvas que favorecem a queda das árvores. Neste período de maior escassez de forragem há possibilidade de consumo de doses maiores de folhas desta arvore, demonstrando que a fome é um importante fator predisponente na intoxicação por P. emarginatus.

As menores doses de $P$. emarginatus que reproduziram o quadro clínico e patológico similar aos casos naturais foram de $20 \mathrm{~g} / \mathrm{kg}$ com folhas verdes em ovinos e de $3 \mathrm{~g} / \mathrm{kg}$ das folhas dessecadas (equivalente $6 \mathrm{~g} / \mathrm{kg}$ folhas verdes) em bovinos. No entanto, bovinos que consumiram doses maiores que 20 e $25 \mathrm{~g} / \mathrm{kg}$ recuperaram-se após demonstrarem alterações clínicas. Estas variações indicam necessidade de melhores estudos para caracterização experimental da toxicidade dessa planta, relativos à sua distribuição geográfica e fase vegetativa.

A evolução clínica observada nos casos naturais e experimentais da intoxicação por Pterodon emarginatus é semelhante à evolução clínica descrita na intoxicação por plantas que causam necrose hepática como Xanthium cavanilessi com evolução clínica de 5 a 72 horas (Colodel et al. 2000), intoxicação por Trema micrantha com evolução de 16 a 67 horas e a intoxicação por Vernonia rubricaulis (Brum et al. 2002) e difere da evolução clínica da intoxicação por Cestrum intermedium que teve evolução que variou de 6 a 12 horas (Bandarra et al. 2008).

As principais lesões observadas nos bovinos dos surtos naturais e dos ovinos e bovinos da reprodução experimental foram semelhantes às lesões descritas em intoxicações por outras plantas hepatotoxicas agudas (Santos et al 2008). Outro achado histológico comum em todos os bovinos dos surtos espontâneos e em ovinos da reprodução experimental foi a presença de células espumosas multinu- cleadas e pericolangite mononuclear. Esse achado é comumente observado em casos de intoxicação por Brachiaria $\mathrm{sp}$, relacionado às saponinas esteroidais litogênicas, que se acumulam em hepatócitos formando cristais (Brum et al. 2007, Brum et al. 2009, Riet-Correa et al. 2011). Os bovinos e ovinos, tanto os que morreram nos surtos espontâneos quanto os que morreram nos experimentos, pastoreavam em piquetes com Brachiaria sp.; entretanto Pterodon emarginatus também contém saponinas esteroídais em suas folhas (Bustamante et al. 2010). Sendo assim, novas investigações precisam ser desenvolvidas para caracterizar se a presença das lesões acima descritas tem relação com o consumo de P. emarginatus.

Agradecimentos.- Ao Instituto Nacional de Pesquisa Científica e Tecnológica (CNPq, Proc.573534/2008-0) pelo suporte financeiro, aos médicos veterinários Dr. Marcos Murilo Rolim Júnior e Dr. Fábio de Souza pelos encaminhamentos das amostras e aos Veterinários do Indea-MT e Iagro-MS que colaboraram com informações epidemiológicas.

\section{REFERÊNCIAS}

Arriaga A.M.C., Castro M.A.B., Silveira E.R., Braz-Filho R. 2000. Further diterpenoids isolated from Pterodon polygalaeflorus. J. Braz. Chem. Soc. 11(2):187-190.

Bandarra P.M., Bezerra Júnior P.S., Corrêa A.M.R., Pedroso M.C., Raymundo D.L. \& Driemeier D. 2008. Intoxicação natural por Cetrum intermedium em bovinos no Rio Grande do Sul, Brasil. Ciência Rural 39(1):262-265.

Barros C.S.L., Catilhos L.M.L., Rissi D.R., Kommers G.D. \& Rech R.R. 2007. Biopsia hepática no diagnostico de intoxicação por Senecio brasiliensis (Asteraceae) em bovinos. Pesq. Vet. Bras. 27(1):53-60.

Bustamante K.G.L., Lima A.D.F., Soares M.L., Fiuza T.S., Tresvenzol L.M.F., Bara M.T.F., Pimenta F.C. \& Paula J.R. 2010. Avaliação da atividade antimicrobiana do extrato etanólico bruto da casca da sucupira branca (Pterodon emarginatus Vogel), Fabaceae. Revta Bras. Pl. Med. 12(3):341-345.

Braga M.M., Castilhos L.M.L. \& Santos M.N. 1985. Biópsia hepática em bovinos: proposta de nova técnica. Revta Centro. Ciênc. Rurais, Santa Maria, 15:79-88.

Braz-Filho R., Gottlieb O.R. \& Assumpção R.M.V. 1971. Chemistry of Brazilian Leguminosae. XXXIV Isoflavones of Pterodon pubescens. Phytochem. 10(11):2835-2836.

Brum K.B., Purisco E., Lemos R.A.A. \& Riet-Correa F. 2002. Intoxicação por Vernonia rubricaulis em bovinos no Mato Grosso do Sul. Pesq. Vet. Bras. 22(3):119-128.

Brum K.B., Haraguchi M., Lemos R.A.A., Riet-Correa F. \& Fioranti M.C.S. 2007. Crystal-associated cholangiopathy in sheep grazing Brachiaria decumbens containing the saponin protodioscin. Pesq. Vet. Bras. 27(1):39-42.

Brum K.B., Haraguchi M., Garutti M.B., Nóbrega F.N., Rosa B. \& Fioranti M.C.S. 2009. Steroidal saponin concentrations in Brachiaria decumbens and $B$. brizantha at diferent develomental stages. Ciência Rural 39(1):279-281.

Carvalho J.C.T., Sertié J.A.A., Barbosa M.V.J., Patrício K.C.M., Caputo L.R.G., Sarti S.J., Ferreira L.P. \& Bastos J.K. 1999. Anti-inflammatory activity of the crude extract from the fruits of Pterodon emarginatus Vog. J. Ethnopharm. 64(2):127-133.

Carvalho J.C.T. 2004. Sucupira, Pterodon emarginatus Vog., p.383. In: Carvalho J.C.T. (Ed.), Fitoterápicos anti-inflamatórios - aspectos químicos, farmacológicos e aplicações terapêuticas. Tecmedd, Ribeirão Preto.

Coelho L.P., Reis P.A., Castro F.L., Gayer C.R.M., Lopes C.S., Silva M.C.C., Sabino K.C.C., Todeschini A.R. \& Coelho M.G.P. 2005. Anti-nociceptive properties of ethanolic extract and fractions of Pterodon pubescens Benth. seeds. J. Ethnopharmacol., 98(1-2):109-116.

Döbereiner J., Tokarnia C.H. \& Purisco E. 1976. Vernonia mollissima, planta tóxica responsável por mortandades de bovinos no sul de Mato Grosso. Pesq. Agropec. Bras., Sér. Vet. 11:49-58. 
Dutra R.C., Braga F.G., Coimbra E.C., Silva A.D. \& Barbosa N.R. 2009a. Antimicrobial and leishmanicidal activities of seeds of Pterodon emarginatus. Braz. J. Pharmacogn. 19(2):429-435.

Fascio M., Mors W., Gilbert B., Mahajan M.B., Monteiro M.B., Santos-Filho D. \& Vichnewski W. 1975 Diterpenoid furans from Pterodon species. Phytochem. 15(1):201-203.

Hansen D. \& Haraguchi M. 2010. Pharmaceutical properties of "sucupira" (Pterodon emarginatus ). Braz. J. Pharm. Sci. 46(4):607-616.

Lemos R.A.A., Guimarães E.B., Carvalho N.M., Nogueira A.PA., Santos B.S., Souza R.I.C., Cardinal S.G. \& Kassab H.O. 2011. Plant poisonings in Mato Grosso do Sul, p.68-72. In: Riet-Correa F., Pfister J., Schild A.L. \& Wierenga T. (Eds), Poisoning by Plants, Mycotoxins, and Related Toxins. Wallingford, UK.

Lorenzi H. 2002. Árvores Brasileiras: manual de identificação e cultivo de plantas arbóreas nativas do Brasil. $4^{\mathrm{a}}$ ed. Instituto Plantarum, Nova Odessa, São Paulo. 368p.

Lorenzi H. \& Matos F.J.A. 2008. Plantas Medicinais no Brasil Nativas e Exóticas. $2^{\text {a }}$ ed. Instituto Plantarum, Nova Odessa, São Paulo. 512p.

Luz Dias F., Takahashi C.S., Sakamoto-Hojo E.T., Vichnewski W. \& Sarti S.J. 1995. Genotoxicity of the natural cercaricides "Sucupira" oil anderemanthine in mammalian cells in vitro and in vivo. Envir. Mol. Mut. 26:338-344.

Machado R.C., Hanusch A.L., Manso J.A.X., da Silva C.C. \& da Cruz A.D. 2011. Investigação da genotoxidade do decocto de Pterodon emarginatus (Fabaceae) em linfócitos t do sangue periférico humano. Anais da $2^{\underline{a}}$ Reunião Brasileira de Citogenética, Águas de Lindóia, SP.
Marques D.D., Machado M.I.L., Carvalho M.G., Meleira L.A. \& Braz-Filho R. 1998. Isoflavonoids and triterpenoids isolated from Pterodon polygalaeflorus. J. Braz. Chem. Soc.. 9(3):295-301.

Purisco E., Lemos R.A.A. \& Salvador S.C. 1998. Intoxicação por Cestrum laevigatum, p.319-321. In: Lemos R.A.A. (Ed.), Principais Enfermidades de Bovinos de Corte do Mato Grosso do Sul. Editora UFMS, Campo Grande, MS.

Prophet E.B., Mills B., Arrington J.B. \& Sobin L.H. 1992. Laboratory Methods in Histotechnology, American Registry Pathology, Armed Forces Institute of Pathology, Washinton, DC. 279p.

Riet-Correa B., Castro M.B., Lemos R.A.A., Riet-Correa G., Mustafa V. \& Riet-Correa F. 2011. Brachiaria spp. poisoning of ruminants in Brazil. Pesq. Vet. Bras. 31(3):183-192.

Sabino K.C.C., Gayer C.R.M., Vaz L.C.A., Santos L.RL., Felzenszwalb I. \& Coelho M.G.P. 1999b. In vitro and in vivo toxicological study of the Pterodon pubescens seed oil. Toxicology Letters 108(1):27-35.

Santos A.P., Zatta D.T., Moraes W.F., Bara M.T.F., Ferri P.H., Silva M.R.R. \& Paula J.R. 2010. Composição química, atividade antimicrobiana do óleo essencial e ocorrência de esteróides nas folhas de Pterodon emarginatus Vogel, Fabaceae. Revta Bras. Farm. 20(6):891-896.

Santos J.C.A., Riet- Correa F., Simões S.V.D. \& Barros C.S.L. 2008. Patogênese, sinais clínicos e patologia das doenças causadas por plantas hepatotóxicas em ruminates e equinos no Brasil. Pesq. Vet. Bras. 28(1):1-14.

Tokarnia C.H., Döbereiner J. \& Peixoto P.V. 2000. Parte Geral, p.3-16. In: Ibid. (Eds), Plantas Tóxicas do Brasil. Editora Helianthus, Rio de Janeiro. 